\title{
El bien como objeto del querer. Suárez y los presupuestos apetitivos y cognoscitivos de la acción racional
}

\section{The good as the object of wanting. Suárez on the appetitive and cognitive conditions of rational action}

\author{
ALEJANDRO G. VIGO \\ Universidad de Navarra, Facultad de Filosofía y Letras, Pamplona \\ avigo@unav.es
}

Resumen. En este artículo se discute la caracterización de Suárez del objeto formal del querer, esto es, el bien. El querer, en todas sus formas posibles, se basa en la cognición, sus diversas formas posibles. Pero el punto de partida para querer algo se da en un juicio acerca de su bondad. Lo que se quiere, a su vez, proporciona el punto de partida para la deliberación. La razón de bien (ratio boni) marca la primera transición (es decir, la transición de la cognición al querer), la ratio finis la segunda (es decir, la transición del querer a la deliberación).

Palabras clave: acción; deseo; voluntad; intelecto práctico; razón de bien; razón de fin.

\begin{abstract}
This paper discusses Suárez's characterization of the formal object of wanting, i.e. the good. Wanting, in all its possible forms, is based on cognition, in its various possible forms. But the starting point for wanting something is given in a judgement about its goodness. What is wanted, in its turn, provides the starting point for deliberation. The ratio boni marks the first transtion (i.e. the transtition from cognition to wanting), the ratio finis the second one (i.e. the transition from wanting to deliberation).
\end{abstract}

Keywords: action; desire; will; practical intellect; ratio boni; ratio finis. 


\section{Introducción}

Lo que voy a intentar ofrecer en el presente trabajo es un intento de profundización de algunos motivos centrales de una interpretación de la concepción suareciana de la acción racional, en atención a los aspectos que tienen que ver tanto con su motivación como con su justificación, que he presentado en trabajos anteriores (Vigo 2011a, 2016). En esos trabajos he procurado mostrar que una adecuada reconstrucción de la posición suareciana, sobre todo, allí donde se trata de caracterizar de modo preciso las contribuciones específicas del intelecto y el apetito -y, más específicamente, de la voluntad como su especie propiamente racional- en el proceso de producción de la acción y en el origen de su cualidad moral, debe orientarse básicamente a partir de la concepción presentada en De anima (en adelante, $D A$ ), y no, por caso, a partir del modo en que Suárez caracteriza la ley en el tratado De legibus, como suele hacerse aún hoy con llamativa frecuencia. A mi modo de ver, este último tipo de enfoque, metodológicamente erróneo, es el que ha conducido reiteradamente a una inadecuada caracterización de la concepción suareciana como una cierta forma de "voluntarismo", un rótulo que se emplea casi siempre sin mayor aclaración de su significado preciso, pero no por ello sin un inocultable tono de reproche, a veces, incluso bastante airado.

Por mi parte, he intentado reconstruir la posición de Súarez por referencia a tres tesis fundamentales, que dan lugar a un sutil balance, a la hora de poner los acentos tanto en los presupuestos apetitivos como en los presupuestos cognoscitivos que dan cuenta de la posibilidad de la acción racional. Las tres tesis mencionadas son las que he denominado: 1) la tesis del primado representacional del intelecto, 2) la tesis del primado motivacional del deseo (vgr. el querer o el apetito, en sus diversas formas), y 3) la tesis del intelectualismo de la justificación. La tesis 1) da cuenta de la dependencia del apetito, en todas sus formas, respecto del acceso a las propiedades descriptivas de los objetos facilitado por las diferentes capacidades cognoscitivas y, en particular, por el intelecto. La tesis 2) da cuenta del hecho de que el mero reconocimiento intelectual de la bondad 
o conveniencia de algo no basta, por sí solo, para motivar la acción correspondiente, sino que debe añadirse, en cada caso, una intención de carácter apetitivo, en la línea de lo que establece el famoso principio aristotélico de que el intelecto, por sí solo, no mueve a nada. La tesis 3), por último, da cuenta del hecho de que la cualidad de la acción viene determinada por su congruencia o falta de congruencia con determinados patrones de enjuiciamiento racional, en conformidad con la regla que establece, en cada caso, la recta ratio. Así reconstruida, la concepción suareciana combina una gama de elementos que apuntan a dar cuenta del aporte imprescindible que realizan tanto la facultad cognoscitiva como la apetitiva, a la hora de hacer posible la acción racional, con su peculiar estructura, que involucra también, y necesariamente, su cualidad moral.

Desde luego, en lo que sigue no voy a reiterar simplemente el análisis ya realizado, sino que me propongo, más bien, precisar un poco más los alcances del modo en el cual Suárez piensa la relación entre la facultad cognoscitiva, en particular, el intelecto, y la facultad apetitiva, en particular, la voluntad como apetito racional, a la luz de lo que implican las tesis 1) y 2). Dicho de otro modo: intentaré precisar un poco mejor el modo en el que Suárez caracteriza el objeto formal del querer, esto es, el bien, a la luz de la constatación elemental de que no hay un acceso del querer, en ninguna de sus formas, a su objeto, que no venga mediado por (los contenidos representacionales ofrecidos por) las diversas funciones cognoscitivas. Lo haré así con vistas a determinar también lo que aporta específicamente el añadido de la intención apetitiva a aquello que, considerado como bueno, adquiere el carácter de objeto querido, vale decir, deseado, apetecido. Me situaré, pues, predominantemente en la zona de confluencia de conocimiento y querer, de intelecto y apetito (voluntad), y tomaré la noción de bien en su sentido puramente formal, que no distingue todavía criteriológicamente entre el bien real y el bien aparente, para usar la terminología que remonta a Platón y Aristóteles. Esto implica no poner aquí en el centro de la atención la tesis 3). Por lo mismo, la discusión estará situada predominantemente en el campo de la teoría de la acción, y no en el de la ética normativa, aunque tematizará también algunos presupuestos comunes a ambas. 


\section{Intelecto y apetito}

\subsection{Intelecto práctico}

Dentro del marco de la discusión de las potencias cognoscitivas llevada a cabo en la la "Disputatio nona" de $D A$, Suárez discute de modo específico la distinción del intelecto teórico (especulativo) y el intelecto práctico en la Quaestio 9. Aquí Suárez reconstruye de un modo poco menos que exhaustivo todos los aspectos relevantes para el establecimiento de dicha distinción. El contexto general del tratamiento, que viene dado por una teoría de las facultades o potencias del alma, explica que buena parte de la discusión esté dedicada a cuestiones como la de si, en el caso del intelecto teórico y el práctico, se trata realmente de dos facultades diferentes o no, y en qué sentido. Pero Suárez realiza, además, toda una serie de otras consideraciones referidas de modo más específico a las funciones del intelecto práctico, que son las que interesan mayormente aquí. Naturalmente, Suárez tiene todo el tiempo en la mira, ante todo, el modo en el que Aristóteles caracteriza la operación propia del intelecto práctico, por contraste con la propia del intelecto teórico. Por cierto, en el tratamiento de Suárez falta toda referencia expresa a la noción aristotélica de verdad práctica, lo cual no puede dejar de llamar la atención. Pero, por otro lado, hay referencias múltiples a las nociones de "conocimiento práctico" (cognitio practica) y "ciencia práctica" (scientia practica).

En un primer paso, Suárez presenta la distinción entre ambos intelectos como una distinción que concierne al fin de la correspondiente operación, a saber: mientras que en el caso de la actividad teórica (especulativa) el fin es la verdad, en la cual el intelecto alcanza su propia perfección, en el caso del uso práctico del intelecto el fin, en cambio, es la obra (opus), que se alcanza por medio de la operación, y no la propia actividad del intelecto, que aparece así subordinada a otra cosa (cf. IX, 9, 2). Cuando habla de la "obra” como fin del intelecto práctico, Suárez se refiere evidentemente al producto mismo o bien la acción misma cuya producción el uso práctico del intelecto guía. Sin embargo, al no hacer referencia expresa a la verdad práctica, en el tratamiento de $D A$ Suárez no está en condiciones de fijar la 
verdad, en general (i.e. en sus dos posibles formas, teórica y práctica), como el objeto formal del intelecto, aunque en el tratamiento del trascendental verum de Disputationes Metaphysicae (en adelante, $D M$ ) dicha estrategia está asumida y claramente delineada (véase $D M$ VIII).

Más bien, Suárez enfatiza aquí el contraste entre el uso teórico (especulativo) del intelecto, cuyo fin es el conocimiento de la verdad, por un lado, y el uso práctico, que apunta como fin a dirigir y regular (dirigere et regulare) la acción humana, por el otro (cf. DA IX, 9, 3). Esta diferencia de finalidad explica también por qué razón es característica del intelecto práctico y las virtudes que lo guían, en particular, la prudencia, la orientación hacia el ámbito de lo particular, pues es allí donde debe realizarse y alcanzar su objetivo la acción humana, mientras que el uso teórico (especulativo) sigue, más bien, la dirección que lleva hacia lo más universal y necesario (cf. IX, 9, 4-5). En efecto, el intelecto práctico opera fundamentalmente con vistas a lo particular y contingente, justamente, en la medida en que pretende orientar y regular la acción, que no sólo se desarrolla en el ámbito de lo contingente, sino que, además, apunta a producir determinados estados de cosas en ese mismo ámbito (cf. IX, 9, 5). ${ }^{1}$ En tal sentido, explica Suárez, el objeto formal del uso teórico (especulativo) del intelecto es la verdad, como tal (veritas ut sic), mientras que el objeto formal del uso práctico es lo que puede ser operado o hecho, como tal (operabile ut sic) (cf. IX, 9, 6).

\subsection{Intelecto y apetito: primado representacional vs. primado motivacional}

En la misma medida en que en su operación apunta a algo diferente de sí mismo, que se aparece como un fin y, en cuanto tal, como algo deseado o apetecido, el intelecto práctico aparece él mismo como necesariamente vinculado a aquella facultad por medio de la cual es, en general, posible desear o apetecer, esto es, el apetito y, más precisamente, el apetito racional,

1 De modo análogo caracteriza Suárez la diferencia entre la ciencia teórica (especulativa) (scientia speculativa) y la ciencia práctica (scientia practica), por un lado, y entre el hábito teórico (especulativo) y el hábito práctico, por el otro. Véase $D A$ IX, 9, 7-8. 
es decir, la voluntad. Ahora bien, en lo que concierne a las relaciones entre el intelecto, por un lado, y el apetito y la voluntad, por el otro, la posición de Suárez en el tratamiento de $D A$ es muy matizada.

Ante todo, Suárez rechaza de plano que el intelecto se denomine "práctico" por el supuesto hecho de ser movido por la voluntad. Más bien, ocurre exactamente lo contrario: se llama "práctico" al intelecto, en la medida en que, de suyo, tiende a mover al apetito y a regular su acto (cf. IX, 9, 9). Suárez intenta conectar esta tesis con lo que Aristóteles señala en De anima III 10, 433a13 ss. Hay que decir, sin embargo, que el pasaje aristotélico no alude en modo alguno al hecho de que el intelecto moviera al apetito, sino que señala algo que apunta, más bien, en una dirección diferente e incluso opuesta, a saber: que el intelecto sólo es práctico allí donde su actividad discursiva tiene lugar “con vistas a alguna otra cosa” (ho heneká tou logizómenos), vale decir, con vistas al fin que es objeto del deseo (órexis). Pero, más allá del hecho de que la cita de Aristóteles no avala lo dicho, Suárez ofrece, además, un argumento independiente en favor de su tesis: el apetito sólo puede dirigirse a lo que es objeto de conocimiento (ad cognitionem), de modo tal que aquello que es el primer conocimiento práctico (prima cognitio practica) debe ser necesariamente anterior a todo acto apetitivo, pues, en caso contrario, se iría al infinito (cf. IX, 9, 9), ya que habría que suponer un conocimiento práctico anterior al que, por hipótesis, se considera que es el primero, y así sucesivamente. Nuevamente, Suárez remite aquí a una supuesta opinión coincidente de Aristóteles, que estaría contenida en Ética a Eudemo, pero el pasaje, cuya ubicación no se indica de modo preciso, es, hasta donde alcanzo a ver, inhallable.

Una explicación posterior permite aclarar un poco mejor lo que Suárez quiere decir: no puede haber apetito, de ningún tipo, sin que esté dado previamente un determinado contenido representacional al cual el apetito se dirige, de modo tal que este último, en su carácter elicitivo, es decir, en su capacidad de dar lugar de modo inmediato a los correspondientes actos de apetencia, se origina a partir del conocimiento (oritur ex cognitione) (cf. X, 3, 11). Aristóteles seguramente no negaría que el deseo presupone necesariamente un componente de índole cognoscitiva, en la medida en que, 
desde el punto de vista de la constitución de su objeto intencional propio, está necesariamente remitido a contenidos representacionales procedentes de las facultades cognoscitivas. Con todo, se buscará en vano en su obra una constatación comparable en su inflexión a la fijación doctrinaria que remonta al aserto de San Agustín según el cual no se puede amar lo que no se conoce. Siguiendo la línea marcada ya por Santo Tomás, Suárez hace suya esta fijación doctrinaria y la incorpora de determinada manera en su propia concepción acerca de la motivación y la producción de la acción. En tal medida, Suárez puede sostener, pues, que el acto del intelecto, como tal, es un presupuesto de la posibilidad del acto del apetito, en el sentido preciso de la tesis del primado representacional del intelecto que he formulado más arriba (= tesis 1): mientras que la actualización de la facultad cognoscitiva se da sobre la base de una unión inmediata de la potencia con su objeto, en el caso de la facultad apetitiva, en cambio, su actualización reclama la unión con la facultad cognoscitiva, a través de la cual se le ofrece el correspondiente objeto (cf. X, 3, 11).

Ahora bien, sería un grave error entender lo anterior como si implicara que el intelecto, a través de sus propios actos, constituye el motor inmediato del apetito. En su tratamiento del apetito, Suárez niega esto terminantemente. La tesis del primado representacional del intelecto es, pues, sólo la mitad de la historia, cuando se trata de dar cuenta del modo en que Suárez intenta explicar la motivación y la producción de la acción. A ella hay que añadirle, además, una tesis complementaria, que he denominado la tesis del primado motivacional del apetito, en general, y de la voluntad, en particular (= tesis 2). Para ilustrarla conviene considerar los siguientes elementos.

En primer lugar, en el tratamiento general de las facultades apetitivas (cf. $D A \mathrm{X}$ ), Suárez se esfuerza por dejar en claro que la dependencia del apetito respecto del intelecto, en lo que concierne al correspondiente contenido representacional, no debe entenderse en el sentido de que el acto del apetito, como tal, fuera causado, sin más, por el acto del intelecto. En cuanto meramente conocido y reconocido como tal, el objeto apetecible no produce, por sí solo, la actualización del apetito, y ello ni en el sentido de hacer actualizar la correspondiente potencia (cf. X, 3, 6), ni tampoco en el 
sentido de concurrir a la esencia del acto propio de la facultad apetitiva (cf.X, $3,7)$. Es la inclinación natural del propio apetito hacia aquello que, en cada caso, se presenta como bueno, y no una supuesta eficacia causal del objeto representado mismo, lo que pone inmediatamente en movimiento a la facultad apetitiva (cf. X, 3, 7). El objeto (re)conocido como apetecible, explica Suárez, no es origen del movimiento del apetito, en el sentido de su causa eficiente inmediata, sino, más bien, término de dicho movimiento, es decir, aquello hacia lo cual tal movimiento tiende o apunta (cf. X, 3, 9). La aprehensión del objeto (re)conocido como apetecible es, pues, necesaria para desencadenar el movimiento del apetito, pero ella misma no podría causar por sí solo dicho movimiento, cuya causa primaria es, pues, la tendencia natural del apetito mismo: es, pues, la inclinación, y no la mera representación, lo que explica el movimiento de la facultad apetitiva (cf. X, 3, 11). En este sentido, Suárez cita el aserto de Santo Tomás, según el cual el intelecto mueve a la voluntad “proponiéndole” (proponendo), vale decir, poniéndole por delante, el objeto al que aquella está naturalmente inclinada (cf. X, 3, 11).

Por otra parte, como lo dejan ver varias aclaraciones de Suárez, el rechazo de la posibilidad de que el acto del entendimiento cause, sin más, el acto de la voluntad se conecta con una serie de aspectos centrales, tanto desde el punto de vista de la psicología y la teoría de la acción, como también desde el punto de vista de la ética normativa. Uno de esos aspectos tiene que ver con el carácter irreductiblemente libre de los actos voluntarios. Suárez explica que sólo Dios puede mover eficientemente la voluntad de modo directo, pero jamás un objeto dado, cualquiera que sea (cf. X, 3, 6). En el tratamiento específico de la voluntad, como forma racional del apetito, Suárez se esfuerza por mostrar que la inclinación natural de la voluntad hacia aquello que el intelecto le presenta como bueno resulta perfectamente compatible con el carácter intrínsecamente libre de los actos voluntarios. Suárez enfatiza que el intelecto no puede imperar elicitivamente la voluntad más que allí donde ya se ha dado un acto previo de la propia voluntad, tal como ocurre cuando, sobre la base del deseo de alcanzar un determinado objeto, el intelecto indica con carácter imperativo los medios conducentes a él (cf. XII, 1, 7). En efecto, el acto de la voluntad se dirige primariamente 
a los fines y sólo secundariamente a los medios, en la medida en que los medios, si no son deseados por sí mismos, sólo aparecen como deseables en cuanto son conducentes al fin (cf. XII, 1, 4). Sin embargo, la constatación relativa a la adecuación de los medios para el fin supone el juicio del intelecto. El carácter libre de la voluntad se relaciona, explica Suárez, con la perfección del intelecto, que es capaz de aislar y considerar separadamente el aspecto de conveniencia de cada cosa y apreciar así su grado de bondad o malicia: la libertad del intelecto para considerar los objetos sensibles bajo diferentes respectos y ponderar así comparativamente sus diferentes propiedades es, pues, la fuente de la libertad de la voluntad, la cual sigue siempre al juicio del intelecto (cf. XII, 2, 10).

Sin embargo, y es un tercer aspecto a tener en cuenta, el rechazo de la posibilidad de que el acto del intelecto mueva de modo directo al apetito no se aplica, a juicio de Suárez, sólo al caso del apetito racional, sino que vale también, de otro modo, para el apetito meramente sensitivo. $\mathrm{Ni}$ en los actos libres ni en los meramente naturales el apetito puede ser eficientemente movido por el objeto de otra facultad (X, 3, 9). Es cierto que el apetito sensitivo se somete a la voluntad, pero lo hace con un tipo de sujeción que Suárez caracteriza como “política”, y no “despótica”, en cuanto no excluye la posibilidad de su oposición al "no" de la voluntad (cf. XI, 3, 2-3). Pero, en rigor, esto último es sólo un modo de hablar. En un pasaje ulterior, siguiendo a Santo Tomás, Suárez precisa de modo mucho más claro cómo debe entenderse la relación de la voluntad y el apetito sensitivo, desde el punto de vista motivacional. Hay que contar aquí con tres posibles escenarios diferentes, a saber: 1) la voluntad puede mover en contra del apetito sensitivo; 2) eliminados otros obstáculos, tales como las alteraciones patológicas o la inmadurez, el apetito sensitivo no puede mover en contra de la voluntad; sin embargo, 3) el apetito sensitivo puede producir por sí solo movimiento, si la voluntad no se opone (cf. XIII, 1, 10).

En suma: puede decirse que el punto de fondo que tiene en vista la concepción de Suárez se refiere al modo en el que el contenido representacional provisto por el intelecto -que en este caso posee, además, un componente de carácter evaluativo- queda incorporado a un circuito intencional-causal 
nuevo, que el intelecto no puede poner en marcha por sí solo, vale decir, si no se añade a él la inclinación propia del apetito. Es éste, el apetito, el que mueve inmediatamente a la acción. En tal sentido, puede hablarse aquí, justamente, de un primado motivacional del apetito. Éste es un punto en el cual la posición de Suárez parece recuperar un aspecto central de la concepción aristotélica de la motivación, pues en su polémica contra el intelectualismo socrático Aristóteles enfatiza que el intelecto, por sí solo, no mueve a nada (Vigo 2011b). La diferencia con Aristóteles reside, paradójicamente, en el hecho de que, situado en la tradición que a través de Santo Tomás se remonta hasta San Agustín, Suárez subraya mucho más fuertemente que el propio Aristóteles los presupuestos cognoscitivos del acto del apetito, en general, y de la voluntad, en particular. Sin embargo, lo hace de un modo que busca evitar, al mismo tiempo, toda recaída en un mero intelectualismo de la motivación, del tipo que fuere. Esta creativa integración de elementos se refleja también, de modo muy similar, en el tratamiento suareciano de la noción de verdad práctica, pues también en este caso Suárez enfatiza de modo particularmente nítido los aspectos referidos a las presuposiciones cognoscitivas que trae consigo la peculiar concordancia con el deseo recto avistada por Aristóteles.

\section{Razón de bien, conveniencia y autorreferencialidad}

Voy a profundizar ahora en el alcance de la tesis del primado representacional del intelecto (= tesis 1), ofreciendo algunas precisiones acerca del tipo específico de juicio del intelecto que subyace a la posibilidad del surgimiento de las diversas formas del apetito. Como se verá, no se trata aquí de un tipo de juicio cualquiera, sino de uno muy específico, a saber: un juicio cognoscitivo, no práctico, pero de alcance evaluativo, que versa sobre el bien, vale decir, sobre la bondad de una determinada cosa. Me limito a unas pocas observaciones destinadas a clarificar los aspectos más importantes del problema.

En primer lugar, hay que recalcar que, a juicio de Suárez, quien sigue en esto una larga tradición que remonta a través de Tomás de Aquino y Agustín hasta Aristóteles y Platón (Sócrates), lo propio del apetito (deseo), en todas 
sus formas, reside en el hecho de que su objeto es siempre intencionado bajo la especie del bien (sub specie/ratione boni), de modo tal que el bien y lo apetecible son, desde el punto de vista puramente formal, lo mismo: el mal, en cuanto mal, no constituye un posible objeto del querer (cf. $D A \mathrm{X}$, $2,3)$. Consiguientemente, en tanto constituye su objeto propio, el bien es al apetito, en general, y a la voluntad, en particular, como la verdad es al intelecto (cf. XII, 1, 1). En tal sentido, que el bien constituye, desde el punto de vista formal, el objeto propio o adecuado del apetito, debe entenderse en el sentido preciso de que todo aquello que es apetecido es apetecido en cuanto se considera, en algún sentido, como bueno. Naturalmente, la noción de bien debe tomarse aquí en un sentido puramente formal, que no prejuzga todavía ni sobre los diversos tipos posibles de apetitos (deseos) y bienes, ni tampoco, lo que es más importante aún, sobre la distinción, crucial desde el punto de vista normativo, entre el bien real y el bien aparente. ${ }^{2}$ En este punto, la formulación de Suárez es lo suficientemente cuidadosa como para prevenir posibles malentendidos: "obiectum adaequatum appetitus est bonum, vel aliqua ratio boni” (cf. X, 2, 3). En este mismo sentido puramente formal está tomada la noción de bien también en el así llamado "primer principio de la razón práctica”, al que Suárez cita en este mismo pasaje y llama "primer principio de la filosofía moral”, a saber: “omne bonum esse prosequendum et malum fugiendum". Tal principio se funda en el simple hecho de que el bien y lo apetecible son una sola cosa, de modo tal que una determinada cosa tiene tanto de apetecible cuanto tiene (o se cree que tiene) de bondad (cf. X, 2, 3: "nam bonum et appetibile unum sunt, et tantum res habet de appetibilitate quantum de bonitate"). ${ }^{3}$

2 Ninguna forma de apetito, ni el natural ni el elicitivo, puede intencionar lo malo bajo la especie del mal (cf. $D A X, 2,3$ ).

3 Que el bien es el objeto adecuado de la capacidad apetitiva no implica que ésta no guarda referencia al mal, sino que queda referida a él de otra manera (cf. $D A \mathrm{X}, 2$, 4). Como ya enfatizó Aristóteles con referencia al caso particular de las potencias racionales, una y la misma potencia se refiere a ambos contrarios, pero no a ambos de la misma manera, sino a uno de ellos como su objeto propio (o natural) y al otro en el modo de la privación. Así, por ejemplo, la medicina tiene por objeto tanto la salud como la enfermedad, pero considera a esta última sólo bajo el aspecto de la privación (stéresis), es decir, como ausencia de la determinación positiva que provee su objeto propio de referencia, en este caso, la 
Ahora bien, aunque, desde el punto de vista formal, el bien constituye el objeto adecuado del apetito, lo cierto es que la aprehensión del bien, como tal, no puede ser operada por la propia capacidad apetitiva. La posibilidad del acceso originario al bien viene necesariamente mediada por determinadas prestaciones cognoscitivas, que, en el caso de los seres vivos no racionales, se basan en la percepción, la memoria y la imaginación, pero que, en el caso del ser humano, involucran también, y necesariamente, la intervención del intelecto, que juzga sobre la bondad de una determinada cosa. No puedo entrar aquí, lamentablemente, en una discusión de detalle relativa al modo en el que Suárez correlaciona las diversas formas de apetito con determinadas prestaciones cognitivas que dan cuenta de la posibilidad de su surgimiento, al facilitar el acceso originario a diversos tipos de bienes o bien de cosas que se presentan como buenas o simplemente parecen serlo.

Como se sabe, en el tratamiento del apetito Suárez sigue la distinción, tradicional desde Platón y Aristóteles, de tres formas básicas, a saber: una forma sensitiva, no racional, la que corresponde a los deseos vinculados con las funciones vitales más básicas (vgr. la epithymía o la "parte concupiscible” del alma); una forma sensitiva, no racional, que, sin embargo, puede seguir u obedecer a la razón, la cual se vincula con la sede originaria de las (ciertas) reacciones emocionales (vgr. el thymós o la "parte irascible" del alma); y una forma racional (vgr. la boúlesis, que en la recepción escolástica corresponde

salud. Véase Aristóteles, Metafísica IX 2, 1046b7-15; véase también Met. IV 2, 1004a9; 1005a4; XI 13, 1061a19). En tal sentido, refiriéndose a la posibilidad de echar mano del arte médico para alcanzar aquello que no es su fin propio o natural, es decir, para enfermar o matar, Aristóteles habla incluso de la existencia de un objetivo o fin según naturaleza (katà phýsin) (p. ej. la salud) y de uno contra naturaleza (parà phýsin) (p. ej. la enfermedad) para cada ciencia (cf. Ética a Eudemo II 10, 1227a25-28). Ahora bien, Suárez parece querer aplicar un modelo, hasta cierto punto, análogo de explicación al caso de las formas puramente sensitivas, es decir, no racionales, del apetito, tal como éstas están presentes no sólo en el ser humano, sino también en otros animales. La razón que hace plausible tal tipo de extensión analógica viene dada, muy probablemente, por el hecho de que toda forma del apetito tiene determinados presupuestos cognoscitivos, de modo que, aunque ninguna forma de apetito meramente sensitivo pueda ser llamada "racional", en sentido propio o pleno, el apetito sensitivo tampoco podría ser reducido, sin más, al nivel de una mera potencia natural, de carácter estrictamente unidireccional, como la que posee el fuego de calentar y quemar, y otras semejantes. 
a la "voluntad"). Suárez discute detalladamente las dos formas sensitivas (no racionales) de apetito en $D A$ XI y la forma racional en $D A$ XII. Esta distribución temática obedece al hecho de que desde un comienzo, vale decir, ya en el tratamiento general de la facultad apetitiva de $D A \mathrm{X}$, Suárez prefiere operar con la distinción básica entre "apetito sensitivo" y "apetito racional”, para luego proceder a establecer distinciones adicionales en el ámbito del primero, y conecta, además, dicha distinción básica con otra estrechamente relacionada, como es la distinción entre "apetito natural" (appetitus naturalis) y “apetito elicitivo” (appetitus elicitivus) (cf. X, 1, 2).

Como señalé ya, no puedo detenerme aquí en la detallada discusión ofrecida por Suárez. Pero me interesa recalcar un aspecto básico. Suárez asume expresamente que en el origen de las diversas formas y los diversos grados del apetito se encuentran diversas formas y diversos grados del conocimiento, de modo tal que cada forma de apetito, por sí sola, no trasciende los límites de lo que ofrece aquella forma específica de conocimiento en la que está fundada. Así, por ejemplo, el apetito sensitivo no trasciende el ámbito abierto por el conocimiento sensitivo (cf. X, 1, 3). Naturalmente, en el caso del ser humano el propio apetito sensitivo queda elevado más allá del plano al que aparece vinculado originalmente, justamente en la medida en que el ser humano está dotado de capacidades intelectuales de enjuiciamiento, que le permiten considerar los bienes a los que quedan referidos los diversos tipos de apetencias sensitivas desde una perspectiva radicalmente ampliada, de modo tal que los juicios evaluativos así resultantes se atienen a patrones de enjuiciamiento que no provienen ya, al menos, no integralmente, de las propias apetencias sensitivas a las que se aplican. Las señales naturales del placer y el dolor, conectadas con las necesidades naturales y su satisfacción o insatisfacción, pierden así, en el contexto fuertemente ampliado de la motivación humana, el carácter de univocidad, que, como indicadores del bien y el mal, poseen en el ámbito de la mera vida animal. Esto explica suficientemente, a mi juicio, por qué razón, con referencia al caso específico de la motivación humana, Suárez sostiene que en el acceso originario al bien, que provee el objeto adecuado del apetito, va siempre involucrado, de uno u otro modo, el juicio del intelecto. 
En efecto, también las inclinaciones naturales y sus objetos, que aparecen conectados con las diversas apetencias sensitivas, deben ser sometidos aquí a un enjuciamiento evaluativo, que ratifique o bien rectifique, en cada caso y en cada contexto particular de decisión y acción, las pretensiones de validez con las que aparecen investidas prima facie, justamente, en la medida en que se presentan naturalmente, es decir, inmediatamente, como bienes, sobre la base del mero conocimiento sensible. ${ }^{4}$

Ahora bien, ¿cómo se debe caracterizar el juicio cognoscitivo-evaluativo del intelecto sobre el bien, desde el punto de vista que atiende a su contenido objetivo? Dicho de otro modo: ¿cómo puede caracterizarse la razón de bien, desde el punto de vista, por así decir, material? ¿Cuál es, propiamente hablando, el contenido objetivo sobre el que recae el juicio cognoscitivo-evaluativo del intelecto, encaminado a establecer si algo ha de considerarse o no como bueno? Para responder a estas preguntas, hay que acudir, siquiera brevísimamente, a la concepción suareciana del bien, tal

4 En el caso de Aristóteles, un buen tratamiento de conjunto de la cuestión relativa a los diversos tipos o niveles de conocimiento vinculados con los diferentes tipos de motivación, no-racional y racional, se encuentra ahora en J. Moss (2012) quien enfatiza que ya la percepción placentera provee una forma elemental y básica de experiencia del bien, de carácter preconceptual, que en la vida animal y también humana juega un papel clave. La prehistoria tendencial de la motivación propiamente racional había sido fuertemente enfatizada ya por M. Riedenauer (2000). El énfasis en los presupuestos tendenciales de la motivación racional no resulta, en modo alguno, incompatible con la asunción suareciana de la tesis del primado representacional del intelecto (= tesis 1 ), puesto que ésta recalca solamente la necesidad irreductible, en el caso de la motivación humana, de la intervención del juicio intelectual sobre lo bueno, a la hora de hacer posible el acceso originario al bien, como objeto del apetito, pero no excluye la posibilidad de que el acto del intelecto tenga, a su vez, presupuestos cognoscitivos y apetitivos más elementales. Lo que queda, en cualquier caso, en pie es el hecho de que en ningún nivel de análisis puede haber algo así como un apetito completamente ciego, pues no puede haber apetito alguno, allí donde no está ya dada alguna forma de percepción, por elemental que ésta sea. Por otro lado, en el caso de la motivación humana, hay formas específicas de apetito que apuntan al conocimiento como a su objeto: los seres humanos tenemos el deseo de conocer, y éste da cuenta incluso de la génesis antropológica de las formas más elevadas de conocimiento, como son las que corresponden a la ciencia metafísica. En su excelente obra sobre la concepción suareciana del derecho, la política y la antropología, el Prof. Coujou ha enfatizado fuertemente este aspecto (véase Coujou 2012, 17-32). Obviamente, esto no excluye que, en su desencadenamiento y despliegue, el deseo metafísico, naturalmente enraizado en el espíritu humano, tenga, a su vez, sus propios presupuestos cognoscitivos. 
como aparece presentada en el marco del tratamiento de los trascendentales en $D M X$.

Lo primero a tener en cuenta aquí es que, aunque coextensivo con el ser, el bien sólo puede aprehenderse sobre la base del acceso cognoscitivo al ser, es decir, sobre la base de la verdad. Dicho de otro modo: sólo sobre la base del correspondiente contenido descriptivo, el cual tiene que ser dado, en cada caso, a través de un determinado acto cognoscitivo, algo puede presentarse como bueno. Nótese, pues, que también en su consideración de los trascendentales Suárez presenta, desde el punto de vista de los correspondientes objetos formales, la misma secuencia que ya estableció en $D A$, desde el punto de vista de las correspondientes facultades: el objeto formal del intelecto, que es la verdad, sigue inmediatamente al ser, pues no es otra cosa que el ser mismo, en cuanto accesible a través de conocimiento, y el objeto formal del querer, el bien, sigue a la verdad, pues sólo puede presentarse y ser considerado (evaluado) como bueno aquello a lo que se tiene ya algún tipo de acceso cognoscitivo, que da lugar, en último término, a un correspondiente juicio cognoscitivo-evaluativo. ${ }^{5}$

Pues bien, en lo que concierne específicamente al contenido objetivo al que queda referido dicho juicio cognoscitivo-evaluativo, hay que recordar que, desde el punto de vista estrictamente metafísico, Suárez caracteriza la bondad trascendental, en general, en términos de "conveniencia" (convenientia) (cf. DMX, 1, 12). En su sentido básico, esta noción no posee, para Suárez, un carácter relacional extrínseco, sino que alude a la perfección intrínseca de una cosa, en tanto dicha perfección constituye, a su vez, el fundamento para toda posible vinculación natural de esa cosa con otras cosas diferentes (cf. X, 1, 12): "ser en sí mismo bueno” y “ser bueno para

5 Obviamente, hay, a su vez, una cierta "prehistoria estética” del juicio cognoscitivo-evaluativo sobre el bien, en la medida en que para Suárez, de acuerdo con la posición habitual de la teoría escolástica del conocimiento, la operación del intelecto se funda, en último término, en el acceso originario a lo real facilitado por la percepción. Pero dejo este punto de lado, porque lo que interesa aquí es el hecho de que, en el caso de la motivación genuinamente humana, la consideración del objeto como algo bueno hace necesaria la intervención del juicio del intelecto. Por otro lado, no hace falta aclarar que lo dicho vale, en esa forma, sólo para contextos en los cuales la formación del juicio cognoscitivo-evaluativo es originaria, vale decir, no se encuentra mediatizada por la habituación. 
otra cosa" configuran, pues, el anverso y el reverso de un mismo fenómeno unitario, que es aquel al que alude centralmente la noción trascendental de bondad (véase Darge 2004, 346-365). Formulado de otra manera: es en virtud de sus mismas propiedades descriptivas, vale decir, de su propio "ser”, como algo puede ser considerado, al mismo tiempo, como un "bien”, y ello tanto en sí mismo como también en relación con otra(s) cosa(s) diferente(s). El acceso a las propiedades descriptivas de una cosa a través del conocimiento constituye, por tanto, un requisito previo para su posible consideración como un cierto bien, y lo que se tiene en vista, cuando se juzga la cosa en cuestión como "buena”, no es sino su "conveniencia”, ya sea en su aspecto básico o intrínseco o bien en su aspecto extrínseco, que está siempre, de una u otra manera, fundado en el primero.

Ahora bien, aunque fundada y en tal sentido derivativa, la conveniencia extrínseca resulta clave a la hora de explicar cómo el objeto juzgado como bueno puede operar como objeto del apetito. En particular, hay que llamar aquí la atención sobre un aspecto no siempre debidamente considerado, a saber: el carácter irreductiblemente autorreferencial que posee el tipo específico juicio cognoscitivo-evaluativo sobre la conveniencia que abre la posibilidad de que lo juzgado como bueno aparezca, al mismo tiempo, como objeto del apetito, es decir, como deseable, en el sentido preciso que remite a la presencia de una genuina intención desiderativa que puede proveer, a su vez, el punto de partida para la operación del intelecto práctico. En efecto, lo que se requiere aquí no es meramente que algo sea considerado como bueno, ya sea en sí mismo o bien por relación a otra cosa, pero de un modo, por así decir, autorreferencialmente desanclado, y situado, como tal, en una perspectiva despojada de involucramiento, como es propio de aquellas constataciones y evaluaciones realizadas desde el punto de vista de la tercera persona, en modo tendencialmente objetivante. En efecto, de tales constataciones y evaluaciones no surge necesaria ni habitualmente deseo alguno. Cuando, siguiendo una larga tradición, Suárez señala que el bien y lo apetecible, y no lo apetecido, son idénticos, elige la terminología muy cuidadosamente, por cuanto no da a entender, de ese modo, que todo aquello que es considerado, en algún respecto, bueno sea, por ello mismo, 
ya inmediatamente apetecido por quien realiza el correspondiente juicio cognoscitivo-evaluativo. ${ }^{6}$ Para que un juicio de tal tipo dé origen efectivamente a una intención desiderativa operante de modo efectivo en el sujeto se requiere, además, que la consideración de la conveniencia no excluya la referencia al propio sujeto de la evaluación. Dicho de otro modo: no basta que algo sea juzgado, en general y en abstracto, como bueno, sino que debe ser considerado, al mismo tiempo, como bueno también para mí, en razón de mis necesidades más comunes, pero también de mis propias características individuales, mi propia situación y las circunstancias concretas en las que debo decidir y actuar.

En tal sentido, Suárez enfatiza expresamente que en todo juicio acerca de la bondad de algo, aún allí donde se trate de un bien ajeno, va involucrada también, y necesariamente, la referencia al propio bien del agente que juzga acerca de dicha bondad: todo bien ajeno se me aparecerá, en algún respecto, también como mi propio bien, si es que realmente ha de contar como algo apetecible para mí (cf. DA X, 2, 7). Este carácter irreductiblemente autorreferencial del tipo específico de juicio cognoscitivo-evaluativo que da lugar a una intención apetitiva operante puede ser explicado, desde el punto de vista que atiende al contenido objetivo del juicio, como fundado en una forma peculiar de conveniencia extrínseca, a saber: aquella que hace referencia al sujeto mismo que juzga sobre lo bueno y, sobre esa base, lo apetece o desea. Obviamente, como no escapa en modo alguno a Suárez, también esta peculiar forma de conveniencia extrínseca está ontológicamente fundada en la conveniencia intrínseca del objeto en cuestión. Más aún: según los casos, puede suponer también formas “objetivas”, vale decir, no autorreferenciales, de la conveniencia extrínseca, como ocurre, por caso, allí donde se desea algo como medio para otra cosa. Así, por ejemplo, son las propiedades de la

6 En rigor, tal como lo ha puesto acertadamente de relieve Darge, en su concepción madura Suárez no incluye la noción de "deseabilidad” (“apetecibilidad”) como parte de la caracterización del bien, en su sentido ontológico-trascendental (véase Darge 2004, 313 ss). Es, más bien, en el contexto práctico donde Suárez se apoya, sin mayores aclaraciones, en la caracterización aristotélica. Pero ello implica, como se verá de inmediato, la puesta en juego de una peculiar noción de conveniencia, dotada de un inflexión más marcadamente “subjetiva”, por así decir, y, con ello, de carácter irreductiblemente "autorreferencial”. 
aspirina las que explican que pueda producir causalmente la desaparición del dolor de cabeza, de modo que si deseo quitarme el dolor de cabeza, puedo desear también tomar una aspirina. Este tipo de caso corresponde a un esquema teleológico causalmente inverso, cuyo origen puede rastrearse hasta Platón (véase Vigo 2007). En el marco de dicho esquema, se tiene que aquello que, desde el punto de vista de la secuencia causal, aparece como efecto es tomado como fin en el acceso de carácter práctico-deliberativo, mientras que aquello que constituye su causa se revela, a su vez, como medio adecuado para la obtención de dicho fin, y ello de modo tal, que la causa, tomada ahora como medio, es deseada indirectamente, justamente, en razón de ser causa de dicho efecto, que, como fin, es deseado por sí mismo. Dicho de modo más económico: si A conduce causalmente a B y B aparece como algo deseable y como un fin que se puede alcanzar a través de la acción, entonces también A aparecerá como algo deseable, al menos, en la medida en que conduce a $\mathrm{B}$, es decir, en cuanto constituye un medio para alcanzar B. La deseabilidad del fin se trasmite así desde éste hacia los correspondientes medios, sobre la base de la conexión causal subyacente.

Pero, más allá de otros aspectos de detalle, el punto que aquí interesa centralmente es el siguiente: a pesar de su carácter necesariamente fundado e incluso doblemente fundado, en el caso del querer que apunta a los medios y otros semejantes, queda en pie el hecho de que la forma “subjetiva” o "autorreferencial” de la conveniencia extrínseca juega un papel clave, a la hora de explicar el verdadero alcance del contenido objetivo de aquel tipo peculiar de juicio cognoscitivo-evaluativo que da cuenta del origen de una intención apetitiva efectivamente operante. En virtud de su carácter irreductiblemente autorreferencial, este peculiar tipo de juicio cognoscitivo-evaluativo se distingue nítidamente de aquellas constataciones y evaluaciones que son llevadas a cabo desde una posición primariamente objetivante y, como tal, tendencialmente despojada del correspondiente componente de autoinvolucramiento ${ }^{7}$. En tal sentido, el lado, por así decir,

7 Esto guarda correspondencia con el tratamiento aristotélico de la producción de la acción en De anima III 9-13 y De motu animalium 6-7, como lo muestra el papel que juegan los procedimientos de indexicalización en primera persona, como mecanismo para dar cuen- 
"objetivo" de la conveniencia, tanto en su dimensión intrínseca como extrínseca, forma parte de las condiciones necesarias, pero no suficientes, de la ocurrencia efectiva del querer. Para que ésta tenga lugar, ha de añadirse, pues, inevitablemente también el requerido componente autorreferencial, que es aquel al que hace referencia específicamente la forma "subjetiva" de la conveniencia extrínseca ${ }^{8}$.

\section{Razón de fin, apetito e intelecto práctico}

Lo dicho hasta aquí permite entender mejor, a mi modo de ver, de qué modo el juicio cognoscitivo-evaluativo del intelecto, en virtud del cual algo es considerado como bueno, más precisamente, como bueno también para el sujeto mismo que juzga, subyace a la generación de la correspondiente intención apetitiva. Tal modo de consideración judicativa de la conveniencia del objeto, también en su aspecto extrínseco de alcance "subjetivo" y "autorreferencial", es lo que constituye en concreto el modo en el cual el intelecto le "propone", le "pone por delante" al apetito, en particular, al apetito racional (voluntad), su objeto propio o adecuado, al cual éste se dirige luego en virtud de su propia inclinación natural, y no por ser movido eficazmente por ninguna otra cosa. A su vez, el objeto apetecido (deseado),

ta de la internalización de los contenidos cognoscitivos que pasan a formar parte de deseos operantes, en el tratamiento del silogismo deliberativo y el silogismo práctico. Para una discusión detallada (véase Vigo 2010). Que la nota distintiva de toda genuina praxis y, con ello, de toda genuina agencia reside en su carácter esencialmente autorreferencial, y no en el componente concomitante de eficacia causal, es a mi modo de ver, una de las intuiciones centrales de la concepción aristotélica de la acción racional (véase Vigo 2008).

8 No hace falta aclarar que todo lo dicho vale también para el caso en el cual el juicio sobre la bondad y la deseabilidad de algo resulta, como tal, materialmente erróneo, pues la noción de bien con la que opera la argumentación desarrollada es, como se dijo ya, puramente formal, en el sentido de que cubre tanto el ámbito del bien real como el del bien aparente, en las diversas formas y especies que puede tener cada uno de ellos. Como es obvio, el componente autorreferencial abre aquí, además, todo un nuevo flanco de posible falibilidad, en la medida en que el agente puede estar también autoengañado respecto de su propio bien real. Se tiene aquí, en una palabra, toda la amplia variedad de posibles errores que amenazan a la praxis humana; a saber: errores respecto de la conveniencia intrínseca, errores respecto de la conveniencia extrínseca objetiva y, por último, también errores respecto de la conveniencia extrínseca de carácter "subjetivo” y “autorreferencial”. 
vale decir, el objeto (considerado) bueno (también para mí), tal como es tenido en la intención apetitiva dirigida a él, provee el punto de referencia para nuevas operaciones intelectivas, que esta vez pertenecen propiamente al uso práctico del intelecto, que, como se dijo al comienzo, es aquel que opera con vistas a algo, es decir, con vistas a un "fin".

Ciertamente, no resulta del todo claro cómo piensa Suárez la vinculación entre la noción de bien y la de fin. Sus declaraciones al respecto son más bien escasas, y quedan un tanto oscurecidas también por los problemas y peculiaridades que caracterizan su tratamiento de la causalidad final, en general. No puedo detenerme aquí a discutir estos aspectos ${ }^{9}$. En todo caso, allí donde se trata específicamente del objeto del apetito y, más precisamente, de la voluntad, Suárez no parece tener demasiadas dificultades en recurrir a la razón de fin, en conexión directa con la razón de bien, afirmando, con Tomás y Aristóteles, la identidad de ambas, en el sentido de que valen ambas siempre, y a la vez, de lo mismo (DMXXIII, 6, 17).

Que el objeto del apetito, tomado como fin, opera como principio del intelecto práctico se afirma expresamente en el tratamiento general del apetito. Vistas las cosas desde la perspectiva que atiende al modo en el que la operación del intelecto práctico depende del acto de la facultad apetitiva, en el sentido de que la averiguación deliberativa de los medios se mueve siempre ya en el espacio de comprensión abierto por la referencia al fin como objeto del apetito (deseo), todo parece indicar que la razón de fin apunta, justamente, a caracterizar específicamente la función que cumple el objeto deseado, en cuanto provee el principio último que da cuenta de la producción de la acción, como obra del intelecto práctico (DA X, 1, 4 ${ }^{\mathrm{a}}$ concl.). Suárez sigue aquí la posición aristotélica que caracteriza al objeto deseado, en tanto fin de la acción, como un motor inmóvil ( $D A \mathrm{X}, 1,6^{\mathrm{a}}$ concl.). ${ }^{10}$ Sobre esta base, parece, pues, posible sugerir que la razón de fin provee el

9 Al igual que en el caso de la "deseabilidad” (“apetitibilidad”), Darge rechaza que Suárez incorpore la razón de fin en su caracterización formal del bien, tomada la noción en su sentido ontológico-trascendental (véase Dargue 2004, 315 s., 361 s., 402 s).

10 Véase también $D A$ XIII, donde se trata brevemente del origen del movimiento locativo voluntario. 
concepto transicional que da cuenta del modo en el cual la operación del intelecto práctico es motivacionalmente dependiente de la del apetito, y ello en el sentido preciso que reclama la tesis del primado motivacional del deseo (= tesis 2).

Por lo demás, el resto de la exposición suareciana resulta, en sus líneas generales, consistente con el modelo explicativo elaborado por Aristóteles. El objeto del apetito, tomado como fin, provee el principio último (primero), de carácter inmóvil, a partir del cual parte o comienza la operación del intelecto, en su uso práctico. En tal sentido, lo primeramente aprehendido opera, a la vez, como principio último de la acción, mientras que todo lo aprehendido posteriormente, es decir, aquello que en su actividad deliberativa el intelecto averigua como medio para la consecución del fin, se sitúa sucesivamente en un tramo cada vez más próximo a la acción misma, hasta llegar al extremo en el cual se encuentra aquello último que opera como su causa próxima, es decir, el medio que puede ser realizado de modo inmediato y que da origen a la cadena de efectos que debe conducir a la consecución del objeto deseado. (DA X, 1). Todo el proceso deliberativo se mueve, pues, en el espacio de comprensión abierto por la referencia al objeto deseado, considerado como fin. Éste, y no otro, es el verdadero alcance de la caracterización aristotélica del intelecto práctico como aquel que calcula o delibera “con vistas a algo", caracterización que, como se vio, Suárez hace suya e interpreta adecuadamente.

\section{A modo de conclusión}

Si la reconstrucción que he ofrecido es en sus líneas generales correcta, hay que decir entonces que el esquema propuesto por Suárez para dar cuenta de las condiciones internas de la producción de la acción comprende, en total, tres tramos vinculados, por así decir, por dos “goznes”. Estos últimos quedan señalizados por sendas nociones transicionales, cuyo empleo apunta a dar cuenta del tránsito, dentro del proceso deliberativo, desde un tramo al tramo inmediatamente siguiente. Dicho de modo esquemático, se tiene, pues, lo siguiente. 
Un primer tramo corresponde a lo que puede llamarse la "prehistoria" cognoscitivo-evaluativa del querer. En este tramo tiene lugar la operación de las facultades cognitivas ( $v g r$. percepción, memoria, imaginación, intelecto) encaminada a la elaboración de un juicio cognoscitivo-evaluativo sobre la bondad (conveniencia) de algo. Aquí hay, por cierto, un contexto motivacional operando ya en el trasfondo, pero no hay todavía una referencia directa a fines, al interior del proceso cognoscitivo mismo. El afán de conocer y evaluar puede estar motivado aquí del modo más diverso, incluso de modos que no tengan ninguna relación intrínseca con los contenidos cognoscitivos considerados y con el juicio cognoscitivo-evaluativo al que conduce finalmente el proceso como un todo. Por lo mismo, el intelecto que aquí opera no es el intelecto práctico o, dicho de otro modo, no tenemos aquí todavía un uso práctico del intelecto, en el sentido estrecho que remite al intelecto que opera con vistas a algo. No se debe confundir, pues, la presencia de interés cognoscitivo, del tipo que fuere, con lo que constituye, en sentido propio, el carácter práctico de un determinado uso del intelecto. No hay uso del intelecto que no esté de algún modo motivado, puesto que también lo está, de diversos modos, cualquier uso meramente teórico-constatativo. Pero la presencia de una cierta motivación no convierte a dicho uso, por sí sola, en un uso práctico, en la medida en que éste no esté él mismo orientado a la “obra”, es decir, a la producción de efectos que hagan posible la consecución de un objeto deseado, diferente del propio conocimiento. Desear conocer y (re)conocer algo como deseable no pueden ser, sin más, identificados.

Un segundo tramo es el que corresponde al surgimiento de un deseo operante o efectivo en el sujeto, sobre la base del juicio cognoscitivo-evaluativo del intelecto que le ofrece o propone el objeto considerado como bueno, es decir, sub ratione boni.

El tercer tramo, por último, es el que corresponde a la operación del intelecto práctico, como tal, que es el que delibera con vistas al objeto deseado, considerado como fin, vale decir, sub ratione finis, aunque Suárez se muestre en ocasiones un tanto reacio a emplear este modo de hablar. Allí donde el proceso correspondiente deliberativo se concluye de modo exitoso, la deliberación da lugar a una decisión (elección) particular, que 
opera entonces como causa inmediata de la acción, es decir, como origen del movimiento (del cuerpo).

En cuanto a los "goznes" que articulan el tránsito del primer al segundo tramo y del segundo al tercero, hay que decir lo siguiente: la consideración del objeto sub ratione boni da cuenta de la transición del primer tramo al segundo, que es la que explica cómo un objeto considerado como bueno se vuelve no sólo apetecible, sino efectivamente apetecido por el sujeto. Por su parte, la consideración del objeto deseado sub ratione finis da cuenta de la transición del segundo tramo al tercero, es decir, del paso del mero deseo de algo al proceso deliberativo que toma el objeto deseado como un fin a alcanzar y, sobre esa base, procede a la averiguación de los medios que hacen posible su consecución.

Por último, si el esquema interpretativo que he propuesto resultara aceptable, habría entonces muy buenas razones para explicar por qué Suárez, por un lado, no incorpora ni la razón de la deseabilidad (apetecibilidad) ni la de fin en la caracterización del bien, cuando éste está tomado en su sentido ontológico-trascendental, pero, por otro, no rechaza ninguna de esas dos caracterizaciones y da indicaciones precisas acerca de su relevancia y el lugar sistemático que les corresponde, allí donde se trata de ofrecer una explicación específica de aquello que constituye el objeto adecuado del querer. Este modo diferenciado de proceder por parte de Suárez no sería, en definitiva, sino una nueva y contundente prueba de su notable agudeza especulativa, y también de su sorprendente lucidez metódica.

\section{Referencias}

Aristoteles. 1831. Aristoteles Graece ex recognitione Immanuelis Bekkeri. Berolini: apud G. Reimerum.

Coujou, J. P. 2012. Droit, anthropologie et politique chez Suarez. Perpignan: Éditions Artège.

Darge, R. 2004. Suárez' transzendentale Seinsauslegung und die Metaphysiktradition. Leiden-Boston: Brill. 
Moss, J. 2012. Aristotle on the Apparent Good. Perception, Phantasia, Thought, and Desire. Oxford : Oxford University Press.

Riedenauer, M. 2000. Orexis und Eupraxia. Ethikbegründung im Streben bei Aristoteles. Würzburg: Königshausen \& Neumann.

Suárez, F. 1961. Disputaciones Metafísicas, edited by S. Rábade. Madrid: Gredos.

Suárez, F. 1991. Commentaria una cum quaestionibus in libros Aristotelis De Anima. Tomo 3, edición crítica por S. Castellote. Madrid: Fundación Xavier Zubiri.

Vigo, A. G. 2007. “Medios y fines en el Gorgias de Platón (466a-468e).” Méthexis (Sankt Augustin) 20: 181-201.

Vigo, A. G. 2008. "Prâxis como modo de ser del hombre. La concepción aristotélica de la acción racional.” En: Filosofía de la Acción. Un análisis histórico-sistemático de la acción y la racionalidad práctica en los clásicos de la filosofía, edited by G. Leyva, 53-86. Madrid: Síntesis.

Vigo, A. G. 2010. “La concepción aristotélica del silogismo práctico. En defensa de una interpretación restrictiva.” Diánoia (México): 65: 3-39.

Vigo, A. G. 2011a. “Intelecto, deseo y razón según Francisco Suárez.” En Razón práctica y derecho. Cuestiones filosófico-jurídicas en el Siglo de Oro español, edited by J. Cruz Cruz, 11-29. Pamplona: Eunsa.

Vigo, A. G. 2011b. “Incontinencia, carácter y razón según Aristóteles.” En Estudios Aristotélicos, editado por A. G. Vigo, 325-362. Pamplona: Eunsa.

Vigo, A. G. 2016. “Intellekt, Wunsch und Handlung. Handlungsproduktion und Handlungsrechtfertigung bei Francisco Suárez.” En The Concept of Law (lex) in Moral and Political Thought of the "School of Salamanca", edited by K. Bunge, M. J. Fuchs, D. Simmermacher, A. Spindler, 229-248. Leiden: Brill.

Vigo, A. G. 2016. “Suárez y la estructura de la verdad práctica” (inédito). 